\title{
La educación física desde la perspectiva de los estudiantes. Un estudio de caso
}

\section{Physical education class from a perspective of students. A case study}

\author{
* Sarita Moreno Pérez, ${ }^{* *}$ Diego Armando López López, \& \\ *** Beatriz Elena Chaverra Fernández
}

\begin{abstract}
Moreno, S., López, D., \& Chaverra, B. (2021). La educación física desde la perspectiva de los estudiantes. Un estudio de caso. Revista
Ciencias de la Actividad Física UCM, 22(2), julio-diciembre, 1-12. http://doi.org/10.29035/rcaf.22.2.7
\end{abstract}

\section{RESUMEN}

La percepción de los estudiantes hacia la clase de Educación Física es de suma importancia para entender cómo mejorarla y lo que representa para ellos. En esta investigación se analizaron las percepciones de los estudiantes frente a la clase, sus preferencias, la importancia que le otorgan y el papel que juega el profesor en su gusto por la Educación Física. La metodología de la investigación fue cualitativa, específicamente un estudio de caso. Se utilizaron como estrategias de recolección de información el cuestionario y el buzón. La investigación se llevó a cabo en una institución educativa públicoprivada de Medellín (Colombia), con la participación de 43 estudiantes de décimo grado (15 y 17 años). Entre los principales hallazgos surgió que a la mayoría de los estudiantes les gusta la clase de Educación Física. Destacan su gusto por clases lúdicas y dinámicas con contenidos deportivos. Entre lo que más les gusta del profesor son sus cualidades: respetuoso, responsable, estricto y amable. Finalmente, le atribuyen la mejora de la salud, el estado físico, el conocimiento y funcionamiento de su propio cuerpo como los fines principales de la clase de Educación Física.

Palabras clave: Educación Física, enseñanza secundaria, método de enseñanza, estudiante de secundaria, estudio de caso.

\section{ABSTRACT}

the class will improve and what it represents for them. In this research, it was analyzed the perceptions and preferences of students about their classes, the importance they give to Physical Education and the role that the teacher has in their liking for it. The research methodology was qualitative, specifically a case study. The questionnaire and the mailbox were used as information gathering strategies. The research was carried out in a public-private high school in Medellín (Colombia) with 43 students between 15 and 17 years old. Among the main findings, it was found that the majority of students like Physical Education class. They like playful and dynamic classes with sports content stands out. Among what they like most about the teacher are his qualities: respectful, responsible, strict and kind. Finally, students attribute to class the improvement of health, physical condition, knowledge and functioning of their own body as the main purposes of the Physical Education class.

Key words: Physical education, secondary education, teaching methods, secondary school students, case studies.

\footnotetext{
* Licenciada en Educación Física, Universidad de Antioquía, Medellín, Colombia. sarita.moreno@udea.edu.co | https://orcid.org/0000-0001-8464-111X

** Licenciado en Educación Física, Universidad de Antioquía, Medellín, Colombia.

diego.lopez4@udea.edu.co | https://orcid.org/0000-0001-8012-1192

*** Doctor en Ciencias de la Actividad Física y del Deporte. Universidad de Antioquía, Medellín, Colombia. beatriz.chaverra@ufrontera.cl | https://orcid.org/0000-0002-1183-8488
} 


\section{INTRODUCCIÓN}

La relación entre los actores educativos y el proceso de enseñanza-aprendizaje puede ser diferente según la asignatura, en consecuencia, la percepción que el estudiantado tenga sobre cada una de estas puede cambiar según la afinidad del estudiantado con su profesor y el contenido. Como lo menciona Blández (2001), es fácil llegar a la percepción del profesor, pero no es igual con los estudiantes debido a diversos factores, el principal, es que saber lo que piensa el estudiante sobre el profesor y los contenidos, puede influir negativamente en las relaciones entre los actores educativos y, por ende, afectar el proceso académico.

El interés por saber cómo perciben los estudiantes las clases de Educación Física, ha sido objeto de estudio por diversos autores en múltiples contextos (Arantes et al., 2018; Beltrán \& Devís, 2019; Cárcamo et al., 2017, Daniel \& Sousa, 2010, Dochy et al., 2002, Gaviria \& Castejón, 2016, Kawashima, 2012, Murcia, 2007, Phillips, et al., 2020). Algunos temas abordados están referidos al profesor, el gusto por la clase, la motivación, el proceso didáctico (metodología, evaluación, recursos, contenidos), entre otros. Sin embargo, conocer qué piensan los estudiantes sobre la clase continúa siendo una pregunta que no se agota, sino que, según los contextos, adquiere mayor relevancia y precisa continuar su profundización.

Comprender qué pasa dentro de la clase y dar importancia a las percepciones de los estudiantes, puede llevar a generar otras maneras de abordar el área, tal vez, con una orientación más integral, formando seres conscientes de su cuerpo, de lo que pueden hacer con él y cómo aportar a todas las dimensiones del ser humano. Conocer estas percepciones permite una reflexión constante para mejorar la enseñanza, nos acercan a saber qué puede ser mejor en las clases de acuerdo a sus respuestas; cómo se puede atraer el interés del estudiante, qué contenidos esperan, que metodologías los acercan al conocimiento, etc., todo con el fin de lograr un aprendizaje significativo.
Al indagar en investigaciones sobre el pensamiento del estudiante, es reiterado el uso del término percepciones. En esta investigación, se asumirán las percepciones como un producto de procesos psicológicos "en los que están implicados el significado, las relaciones, el contexto, el juicio, la experiencia pasada y la memoria" (Schiffman, 2004, p. 2). En este sentido, queremos conocer qué piensan los estudiantes sobre la clase de Educación Física, cómo la han vivido y qué proponen para mejorarla.

Entre algunos estudios que abordaron las percepciones de los estudiantes encontramos a Blández (2001), quien alude que la comprensión de las perspectivas del alumno sobre la enseñanza es tan importante para entender los encuentros en la clase, como lo es la construcción social que los profesores tienen de sus alumnos. Además, es fundamental entender la forma en que los estudiantes definen la situación dentro de las clases, debido a que es el único modo en que se puede dar sentido a sus acciones.

Por su parte, Murcia \& Jaramillo (2005) afirman que la clase de Educación Física reproduce dinámicas propias de sesiones de entrenamiento deportivo volviéndose monótonas debido a sus contenidos deportivizados y a sus métodos poco participativos y dinámicos donde no se satisfacen las necesidades motrices y de socialización que los jóvenes necesitan. Algunos años después Murcia (2007), describe estas percepciones de los estudiantes como imaginarios institucionalizados e imaginarios radicales. Los imaginarios institucionalizados son el esbozo de las estructuras del deporte y los imaginarios radicales son esa posibilidad que tienen los jóvenes para ser ellos mismos, para encontrarse con el otro y lo otro, para escapar de las realidades normatizadas de la vida escolar.

Gaviria \& Castejón (2016), encontraron que la percepción del estudiantado sobre lo que no les gusta de la clase de Educación Física es la monotonía y la poca oferta de contenidos. Otros estudios también encontraron que los estudiantes 
destacan la metodología como uno de los elementos que más les gusta de la clase de Educación Física, mencionando que las clases prácticas son la forma en que ellos consideran que se debe impartir la asignatura, para su diversión y entender diferentes temas a través del movimiento (Blández, 2001, Gaviria, 2016).

De acuerdo a los estudios anteriores y reconociendo las múltiple preguntas que aún se pueden abordar sobre el pensamiento del estudiante hacia la clase, esta investigación tuvo como objetivo comprender las percepciones de los estudiantes de una Institución Educativa de Medellín-Colombia sobre la clase de Educación Física, y por medio de ello dilucidar qué se puede fortalecer en el desempeño educativo de los estudiantes, mejorar la manera de compartir los conocimientos por parte del profesor y fortalecer el ambiente de clase.

\section{METODOLOGÍA}

La investigación cualitativa fue el método escogido para este estudio, dado el interés por entender los fenómenos sociales desde la propia perspectiva y palabras de los participantes (Taylor \& Bogdan, 1987). En este sentido, el método cualitativo fue determinante para conocer las situaciones, vivencias y pensamientos que experimentan los estudiantes en las clases de Educación Física. Al ser un diseño abierto, emergente y flexible (Galeano, 2004), permitió ajustar el proceso investigativo a las realidades y circunstancias del contexto, sin olvidar los criterios de rigor del estudio.

Se asumió como enfoque el estudio de caso descriptivo e interpretativo con un objetivo instrumental (Merriam, 1988, Yin, 1984), pretendiendo comprender a profundidad las percepciones de los estudiantes sobre la clase de Educación Física.

La investigación se llevó a cabo en una institución educativa público-privada de la ciudad de Medellín, con la participación de 43 estudiantes (23 hombres y 20 mujeres) de décimo grado de educación media con edades aproximadas entre 15 y 17 años. Este grupo tenía dos clases de Educación Física a la semana de 50 minutos cada una.

Los participantes se seleccionaron con criterios de representatividad cualitativa, no estadística (Galeano, 2004), por lo tanto, se determinaron unos criterios en los términos que proponen Goetz \& Lecompte (1988), lo que implicó que como investigadores determináramos un conjunto de atributos que debían poseer los estudiantes de este estudio. Los criterios fueron: Que fuera uno de los grupos en que dábamos la clase de Educación Física para tener un contacto permanente con ellos; que fueran de los grados superiores, ya que contaban con mayor experiencia en el área por su vivencia en años escolares anteriores y tendrían un lenguaje más elaborado para comprender lo que manifestaban desde sus perspectivas.

Las estrategias de recolección de información fueron el cuestionario y el buzón. El cuestionario es una estrategia que permite medir la forma en que las personas encuestadas poseen determinados conceptos de interés (opiniones, creencias, conductas, etc.) (Canales, 2006). Este instrumento permitió conocer las apreciaciones de los estudiantes frente a lo que vivían en la cotidianidad de las clases de Educación Física. Se utilizaron preguntas cerradas con varias opciones de respuesta y preguntas abiertas para la libre expresión (Piéron, 1999). Para esta investigación se utilizó el cuestionario denominado "el pensamiento de los estudiantes sobre la asignatura de Educación Física" utilizado en la tesis doctoral de Gaviria (2016).

El buzón, se utilizó como una estrategia abierta y permanente en los términos de Santos (1993). Este instrumento permitió recoger ideas, sentimientos y percepciones que tal vez no podían expresarse libremente en otras circunstancias. Esta estrategia fue utilizada en un estudio anterior (Blández, 2001) con un propósito similar a esta investigación. En el buzón, los estudiantes introducían escritos anónimos, resultado de preguntas acerca de su percepción sobre la clase de Educación Física, estas 
cartas las podían realizar al finalizar la clase o durante cualquier momento de la jornada escolar.

Este estudio requirió en todo momento de la triangulación de métodos, permitiendo ampliar y profundizar la comprensión de la información obtenida por las diferentes estrategias (Okuda \& Gómez-Restrepo, 2005) con la construcción teórica y de investigaciones previas. El análisis de la información fue realizado de forma constante e intensiva. Basados en Taylor \& Bogdan (1987) se tuvieron en cuenta las tres fases que proponen así:

a) Fase de descubrimiento: Se comenzó la búsqueda e identificación de temas provenientes de las respuestas del cuestionario y los escritos del buzón, desarrollando nuestro propio concepto, reflexiones y proposiciones sobre las categorías que emergieron. Para esto, partimos de una búsqueda de material bibliográfico y lectura de estudios previos que nos permitieron interpretar la información.

b) Fase de codificación: se desarrolló la interpretación de la información de modo sistemático, permitiendo refinar, expandir o descartar ideas iniciales, es decir, se realizó una codificación abierta Strauss \& Corbin (2002) o inductiva (Bonilla \& Rodríguez, 1997). En el estudio emergieron tres grandes categorías, que a su vez, contienen subcategorías que ayudan a comprender la percepción que tienen los estudiantes sobre la asignatura de Educación Física, estas son: el proceso didáctico, el profesor y los fines de la Educación Física (Figura 1).
En esta fase también se definieron los códigos que permitieron organizar la información recopilada, y a la vez proporcionar el anonimato. Los códigos definidos fueron: para cuestionario C.E. que significa cuestionario estudiante y el número, al igual el buzón con B.E que significa buzón estudiante y el número asignado a cada estudiante.

c) Fase de relativización de los datos: Por último, se trató de interpretar la información en el contexto en el que fueron recogidos.

Entre las consideraciones éticas se tuvo el consentimiento de la institución para realizar la investigación y el asentimiento informado para los padres o representantes legales de los estudiantes, en ambos documentos se informó el propósito del estudio, las estrategias de recolección de información, la participación voluntaria y anónima y el uso exclusivo de la información obtenida con fines académicos. También fue claro para los estudiantes que sus opiniones no interferirían, ni tendrían influencia en la evaluación dentro de la clase, dejando clara la confidencialidad de la información. Una vez terminado el estudio, se presentaron los hallazgos ante la comunidad educativa.

\section{RESULTADOS Y DISCUSIÓN}

De acuerdo a la metodología elegida y las estrategias utilizadas para la obtención de la información, es pertinente presentar los resultados y la discusión de manera conjunta. 


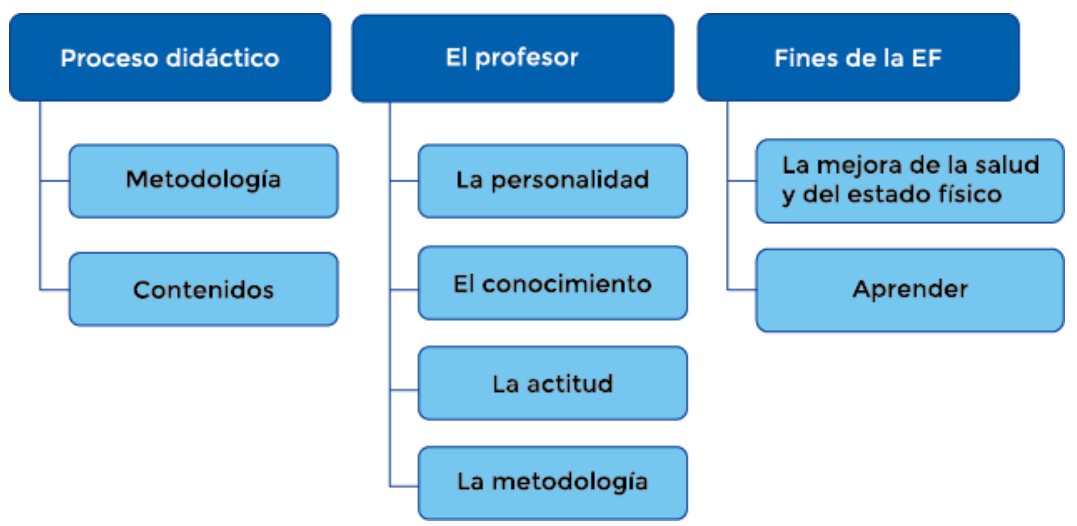

Figura 1. Categorías emergentes.

\section{EL PROCESO DIDÁCTICO}

Al preguntarle a los estudiantes ¿Qué es lo que más les gusta y agrada de la clase Educación Física?, surge el proceso didáctico como el aspecto que más resaltan, específicamente se enfocan en la manera en que se abordan las clases, es decir la metodología. Destacan en sus discursos las actividades realizadas y que es una asignatura en su mayoría práctica que les permite moverse y estar fuera del salón. En este sentido, señalan las clases como lúdicas, dinámicas, con temas diversos, además, que son clases explicadas y orientadas por los profesores de una forma agradable. Respecto a esto, algunos estudiantes dicen:

B.E.21: "Las clases de educación física han sido muy dinámicas y eso me ha gustado mucho, en general las clases han sido muy buenas".

B.E.6: "Me gusta que las clases sean distintas con temas diversos".

B.E.18: "Me gusta cuando las clases son lúdicas, más de juego".

C.E.30: "(Me gustan) Actividades diferentes a las conocidas, como deportes diferentes".

C.E.22: "La variedad en las clases que se realizan".

Algunos estudios concuerdan en afirmar que los estudiantes destacan la metodología como uno de los elementos que más les gusta de la clase de
Educación Física, mencionando que las clases prácticas son la forma en que ellos consideran que se debe impartir la asignatura, para su diversión y para entender diferentes temas a través del movimiento (Baena et al., 2016; Blández, 2001, Gaviria \& Castejón, 2016).

Estos resultados contrastan con las conclusiones del estudio de Phillips et al. (2020), donde los estudiantes señalan las clases como aburridas, y por tanto, es un motivo para disminuir las actitudes positivas hacia la Educación Física.

Por otro lado, los contenidos son otro elemento del proceso didáctico que más les gusta de la clase, específicamente los deportes como fútbol, voleibol y baloncesto, temas que, año tras año han visto en sus clases. Algunos estudiantes también se refirieron al ejercicio físico como un tema agradable. En ese sentido, algunos estudiantes expresaron:

\section{C.E.29: (Me agrada) "Jugar fútbol o voleibol". \\ C.E.16: "El deporte".}

C.E.35: "Que hagamos (ejercicio) físico como resistencia y fuerza".

B.E.8: "Me gustan los temas".

Estos mismos deportes fueron escogidos por el $74,4 \%$ de los estudiantes como los deportes que más les gusta practicar en las clases de Educación Física.

El $72,1 \%$ de los estudiantes, manifestó que les gusta y les gusta mucho la clase de Educación Física, 
confirmando así, que desde los instrumentos de recolección de información sea buzón o cuestionario los resultados tienden a mostrar que la asignatura a la gran mayoría le gusta y le agrada. Este resultado se corrobora con otros estudios donde los estudiantes manifiestan su gusto por la clase de Educación Física (Daniel \& Sousa, 2010, Gaviria, 2016, Moreno \& Hellín, 2007).

Sólo el 27,9 \% de los estudiantes expresó que les gusta poco la clase de Educación Física. Al preguntarles qué es lo que menos les gusta, hacen referencia a la metodología y los contenidos. Cabe resaltar que, aunque son los mismos resultados que encontramos en lo que más les gusta de la clase, se refieren específicamente a las clases teóricas, es decir, a los estudiantes no les gusta la manera en que se imparten estas clases y cómo se abordan los contenidos conceptuales (trabajos escritos, quices y talleres). Así se evidencia en los testimonios.

\section{C.E.4O: "(No me gusta) La manera en que nos ponen unos trabajos".}

C.E.35: "Cuando nos quedamos en el salón haciendo otra cosa que no sea deporte".

B.E.3: "No me gusta mucho que se ponga por delante la teoría antes que la práctica y que la exigencia sea tan grande".

B.E.19: "Los trabajos escritos y los quices son muy aislados del pensamiento de un deportista... A veces tienen preguntas capciosas o retóricas y es injusto, arbitrario e innecesario".

Hallazgos como este, son compartidos con otras investigaciones, donde se ha encontrado que a los estudiantes no les gusta la forma en que se ve la teoría y que prefieren un espacio de liberación de tensiones que les permita dinámicas donde se involucre el movimiento (Blández, 2001, Gaviria \& Castejón, 2016, Jaramillo \& Hurtado, 2006, Murcia, 2007).

Estas expresiones de los estudiantes son un llamado a pensar la manera en que se abordan los elementos teóricos en la clase, dado que el estudiantado tiene una visión más práctica del desarrollo de las clases y al no ser de esta manera, hace que la enseñanza la asuman como las otras áreas, ocasionando una predisposición negativa frente a las clases. En este sentido, es importante conocer su percepción para buscar alternativas didácticas que no vinculen los contenidos conceptuales a sensaciones de apatía y desinterés.

Del mismo modo, encontramos que, respecto a los contenidos, los estudiantes entran en una ambigüedad. Por un lado, un amplio número de estudiantes destacan el deporte como el contenido que más les gusta de la clase, pero a la vez, otro grupo de estudiantes reclaman que estos contenidos se hacen monótonos, es decir, no les gusta practicar siempre los deportes tradicionales (voleibol, fútbol, baloncesto).

C.E.21: "a veces son muy iguales (las clases) y no hay cosas diferentes en el entorno deportivo".

B.E.20: "Me gustaría que la educación física fuera más que sólo deporte".

C.E.22: "(No me gusta) Cuando solo ponen las actividades de siempre como fútbol, voleibol y baloncesto".

B.E 28: "Cuando se ve lo mismo como fútbol, baloncesto y voleibol".

Esta dualidad de gustos por los deportes, también se encontró en el estudio de Blández (2001). Los estudiantes manifiestan la necesidad de abordar los deportes en la clase, sean éstos conocidos o nuevos, no obstante, no se refieren sólo a deportes, sino a otros contenidos más amplios que les permita explorar nuevas alternativas o descubrir nuevos gustos, saliendo de lo rutinario y la desmotivación (Gaviria, 2016, Murcia, 2007, Murcia \& Jaramillo, 2005).

Asimismo, algunos estudiantes manifiestan que les desagrada realizar ejercicios físicos agotadores como planchas, abdominales y trotar. Estudios como el de Moreno \& Hellín (2007), mencionan que mientras haya poco interés, el valor positivo hacia actividades de condición física desaparece. 
Al preguntarles cómo mejorarían la clase, sus respuestas se enfocaron en estos aspectos (clases teóricas y contenidos).

C.E.35: "Yo sé que la teoría es muy necesaria, pero que el profe la explique mientras hacemos alguna actividad".

B.E.9: “... no ser tan cuadriculados en las clases, siempre apegados a una teoría".

C.E.22: "Más variedad en las clases, no solo los deportes de siempre".

B.E.22: "...más actividades con nuevas tendencias deportivas y no siempre los mismos deportes".

Estos testimonios refuerzan la idea que la clase puede mejorarse incorporando deportes tanto tradicionales como nuevas tendencias, esta misma idea se ha encontrado en otros estudios (Blández, 2001; Gaviria, 2016). El deporte como parte de la clase, corrobora la idea que el alumnado piensa en una Educación Física deportivizada muy ligada a la línea tradicional del área (Murcia, 2007).

\section{EL PROFESOR}

En relación a la percepción que tienen los estudiantes sobre la clase, surge también el profesor como una categoría importante de la cual se rescatan cuatro elementos o subcategorías: la personalidad, el conocimiento, la actitud y la metodología que utiliza.

En cuanto a la personalidad, los estudiantes manifiestan que lo que más les gusta del profesor son las cualidades que él posee: respetuoso, responsable, estricto y amable.

B.E.20: "Respetuoso, atento, innovador, responsable".

C.E.12: "Que es estricto".

C.E.25: "Paciente y respetuoso".

C.E.43: "Es muy recto, serio".
El 95,3\% del estudiantado considera que el profesor los trata con respeto, lo que deja en evidencia que es un rasgo de la personalidad del docente muy valorado por sus estudiantes. El resultado, en este caso, es similar a los que han aportado otros estudios (Cárcamo, 2012, Gaviria \& Castejón, 2016, Phillips et al., 2020).

Asimismo, los estudiantes consideran importante el conocimiento que el profesor posee y que les puede aportar a su formación, no solo desde el dominio pleno de los temas, sino también desde su aprovechamiento y ejecución.

C.E.27: "Que tenga un buen conocimiento del área".

B.E.31: “...saber de la materia, y enseñarnos mucho de esta materia".

Esta importancia que otorgan al conocimiento del área por parte del profesor, se confirma cuando el 95,3\% de los estudiantes considera que el profesor domina los contenidos. Otros estudios también han expresado que es importante que el profesor posea un conocimiento y preparación académica especializada dentro del área de Educación Física (Cárcamo, 2012, Moreno \& Hellín, 2007, Murcia, 2007).

Otro elemento que destacan los estudiantes como determinante en el profesor, es la actitud que tenga hacia ellos, dado que esto los motiva y dispone a tener un mayor acercamiento hacia la clase.

B.E.29: "Ser paciente, servicial, además de tener mejor actitud y siempre estar dispuesto a ayudar a los estudiantes".

B.E.15: "Demostrar el interés hacia el rendimiento de los alumnos".

C.E.5: "La motivación".

C.E.21: "Que es atento".

Aquí se encuentran similitudes con el estudio de Hurtado \& Jaramillo (2006) y Sözen \& Korur (2020) donde los estudiantes tienen la idea del profesor como alguien con quien se puede hablar sin problemas dentro de la clase, que brinda confianza y 
ánimo. De igual manera, otros estudios confirman el valor que dan los estudiantes a las actitudes del profesor como su alegría, ecuanimidad, el trato hacia ellos, etc. (Gaviria \& Castejón, 2016, Moreno \& Hellín, 2007).

Aunque la mayoría del estudiantado valoró positivamente la influencia del profesor en su gusto por la clase, encontramos algunos comentarios de los estudiantes que hacen referencia a dos aspectos que no les agradan del profesor: aspectos de la personalidad y decisiones metodológicas. En la personalidad destacan el ser estricto y su carácter serio.

C.E.22: "Cuando es tan estricto en cosas sin importancia, que no afectan en nada el aprendizaje".

C.E.2: "es muy serio".

Este hallazgo se corresponde con los resultados del estudio de Cárcamo (2012) donde los estudiantes resaltan entre las características negativas del profesor, ser muy estricto. Sin embargo, estas percepciones dejan ver una contrariedad en los participantes de esta investigación, dado que 17 alumnos ven como positivo esos mismos rasgos de su personalidad. Lo mismo sucede con estudios donde los estudiantes esperan que el profesor sea exigente con ellos, pero a la vez, desaprueban esa actitud (Blández, 2001, Murcia, 2007).

En el aspecto metodológico, algunos estudiantes mencionan que no les gusta la forma en que se solicitan los trabajos teóricos y las pocas oportunidades de clase libre. Es de resaltar que los estudiantes señalaron que, lo que más les gusta de la clase fue el método del profesor, pero específicamente su manera de abordar las clases prácticas, las actividades y el juego, lo que corrobora esta percepción sobre el poco gusto por los trabajos teóricos.

C.E.4: (No me gusta del profesor) "La forma a veces de poner los trabajos".

C.E.17: "A veces sobrepasa la exigencia con los ejercicios".
B.E 31: "Me gustaría que se hiciera más práctica y menos teórico..."

La idea anterior se corrobora con lo encontrado en el estudio de Moreno \& Hellín (2007), cuando mencionan que el diseño de clases motivantes por parte del profesor genera más gusto por la asignatura de Educación Física. Asimismo, es el profesor quien, a través de ese desarrollo metodológico, influye en la calidad de las clases y genera motivación para realizarlas (Daniel \& Sousa, 2010).

Desde la perspectiva de los estudiantes, un buen profesor de Educación Física debe tener entre sus características esenciales ser innovador, explicar bien y proponer una buena metodología.

B.E.12: "Que sepa utilizar las clases para que no sean aburridas teniendo buena metodología".

C.E.39: "Su manera de dirigir una clase, su metodología".

C.E.22: "Su forma de explicar los temas en la clase de Educación Física".

Asimismo, destacan como cualidades importantes: mantener la disciplina en la clase (74,4\%), el respeto hacia los estudiantes $(69,8 \%)$ e incentivar y ayudar a aquellos que tienen dificultades $(62,8 \%)$.

Es evidente entonces, que el profesor cumple un papel importante en la clase de Educación Física según los valores y el conocimiento que demuestra, ya que es quien motiva a los estudiantes a realizarla, llevando a que se cree un ambiente propicio de aprendizaje y atrae a los estudiantes por su manera de enseñar.

\section{FINES DE LA EDUCACIÓN FÍSICA}

Al preguntarle a los estudiantes ¿Cuál es la importancia de la Educación Física en tu formación integral?, el 86\% del estudiantado, dejó claro que es muy importante e importante la asignatura para ellos, resultados que coinciden con otros estudios (Cárcamo et al., 2017, Gaviria, 2016, Jaramillo \& 
Moreno, S., López, D., \& Chaverra, B. (2021). La educación física desde la perspectiva de los estudiantes. Un estudio de caso. Revista Ciencias de la Actividad Física UCM, 22(2), julio-diciembre, 1-12. http://doi.org/10.29035/rcaf.22.2.7

Hurtado, 2006, Moreno \& Hellín, 2007, Murcia, 2007) Esta percepción la confirman en sus testimonios de buzón y argumentan sus razones.

Estos argumentos, se convierten en los objetivos principales o fines que tiene el área para ellos y se agrupan en dos grandes elementos. El primero es la mejora de la salud y el estado físico, es decir, para los estudiantes participantes de esta investigación, la Educación Física les sirve principalmente para cuidar el cuerpo, mejorar el rendimiento físico y llevar mejores hábitos de vida saludable.

B.E.8: "Es importante para saber el funcionamiento del cuerpo, estar saludable..."

B.E.15: "Nos ayuda a conocer nuestro cuerpo y a tener mejores hábitos para la salud".

B.E.29: "Nos ayuda a mejorar nuestra salud tanto física como mental".

Estos fines que le otorgan los estudiantes al área, se reafirman en el cuestionario cuando un $74,4 \%$ de alumnos expresó que las clases de Educación Física sirven para mejorar la salud y la condición física. Resultados similares tienen otros estudios donde resaltan que, para los estudiantes, la Educación Física es importante porque proporciona una vida más saludable y sirve para mejorar la condición física (Daniel \& Sousa, 2010, Gaviria, 2016)

Esta idea de los estudiantes deja claro que las clases de Educación Física son entendidas desde una perspectiva biológica, dejando una enseñanza integradora fuera de la visión de la Educación Física para ellos. Estas percepciones muestran que la idea de Educación Física deportivizada ha sido enriquecida desde el entorno escolar con los contenidos que han recibido a lo largo de su educación y reforzados en los entornos extracurriculares. En ese sentido, otros trabajos han manifestado resultados similares mostrando que la importancia del área y su utilidad está enfocada en lograr beneficios físicos (Gaviria \& Castejón, 2016, Jaramillo \& Hurtado, 2006)
En segundo lugar, los estudiantes consideran el aprender como otro de los fines esenciales de la Educación Física. Ellos manifiestan que la clase les permite saber cómo funciona el cuerpo, sobre las capacidades que tienen y conocer nuevos temas, destacando la transversalidad de los aprendizajes y la aplicabilidad de esta asignatura para diferentes situaciones que se presentan en su entorno.

C.E.2: (en la clase de Educación Física) "uno aprende cosas nuevas".

B.E.12: "Me gustó demasiado saber sobre los huesos y la parte en que nos conocemos más nosotros".

C.E.27: "Poder aprender el funcionamiento del cuerpo".

B.E.23: "Realmente para en un futuro ayudar a los demás, o simplemente a mímismo."

El 11,6\% de los estudiantes expresaron que la Educación Física les servía para aprender cosas nuevas. Además, el 55,8 \% de los estudiantes, creen que poseen un conocimiento suficiente respecto a la asignatura, lo que corrobora la finalidad de aprendizaje que le confieren al área.

Cabe mencionar que estas respuestas resultan similares a lo expresado por Gaviria (2016), donde los estudiantes tienen objetivos similares a alcanzar en esta asignatura. En contraste, el estudio de Phillips et al., (2020), encontró que muchos estudiantes señalan que en la clase de Educación Física no se aprende, no obstante, no fue claro en el estudio si se debe al profesor u otros factores.

\section{CONCLUSIONES}

Este estudio permitió tener un mayor acercamiento $y$, por tanto, mayor conocimiento, sobre las percepciones de los estudiantes frente a la clase de Educación Física. Tomando como referencia el objetivo de esta investigación y la información obtenida, podemos concluir que a la mayoría de los estudiantes les gusta la clase de Educación Física. Desde su experiencia, ellos tienen la capacidad de identificar en las clases la metodología que usa el 
profesor, expresando su gusto específico hacia clases más lúdicas y dinámicas, además, destacan la importancia y la necesidad que sean bien orientadas y explicadas. Asimismo, la personalidad, el conocimiento, la actitud y la metodología que posee el profesor, son esenciales para que los estudiantes se sientan motivados y participen activamente de las clases.

Comprendemos que si bien surgieron categorías importantes que abarcan las percepciones de los estudiantes, también nos percatamos que en ocasiones el estudiantado no alcanza a visualizar otros aspectos que pretende la clase de Educación Física como el aprendizaje de valores, el trabajo en equipo, la toma de decisiones, la formación de la norma y demás aspectos que se encuentran implícitos en las clases y que son fundamentales e importantes para su formación y crecimiento personal. Tal vez, hace falta un mayor esfuerzo por parte del profesorado para que estos elementos se hagan visibles en los estudiantes.

Para finalizar, reconocemos que conocer lo que piensan los estudiantes permite al profesorado mejorar su enseñanza y, por ende, la visión positiva que puedan construir los estudiantes sobre el área. No obstante, es evidente que, para entender el pensamiento del estudiantado, se deben realizar más estudios que permitan retroalimentar aquello que los maestros realizan en sus clases y así, tener un panorama más amplio que aporte a mejores procesos de formación desde la Educación Física. Cabe resaltar que lo encontrado en esta investigación forma parte de lo que esta población, en este contexto educativo en particular ha ido desarrollando a través de todo su proceso escolar, sin embargo, puede generar inquietudes para otros contextos y motivar a desarrollar otras investigaciones que permitan profundizar en el tema.

\section{REFERENCIAS BIBLIOGRÁFICAS}

Arantes da Costa, L., Flores, P., Andrade, N., Anversa, A., \& Matias de Souza, V. (2018). Tecendo relações entre a motivação para as aulas de educação física e o Ideb. Revista Brasileira de Ciencias do Esporte, 40(4), 370-373. https://doi.org/10.1016/j.rbce.2018.03.018

Baena-Extremera, A., Gómez-López, M., GraneroGallegos, A., \& Martínez-Molina, M. (2016). Modelo de predicción de la satisfacción y diversión en Educación Física a partir de la autonomía y el clima motivacional. Universitas Psychologica, 15(2), 15-25. https://doi.org/10.11744/Javeriana.upsy152.mpsd

Beltrán-Carrillo, V., \& Devís-Devís, J. (2019). El pensamiento del alumnado inactivo sobre sus experiencias negativas en educación física: los discursos del rendimiento, salutismo y masculinidad hegemónica. Revista Internacional de Ciencias del Deporte, 15(55), 20-34. https://doi.org/10.5232/ricyde2019.05502

Blández, J. (2001). Lo que el alumnado de educación secundaria piensa de las clases de educación física y no se atreve a decirle a su profesor/a. Apunts: Educación Física y Deportes, 4(66), 20-30. https://revistaapunts.com/lo-que-el-alumnado-deeducacion-secundaria-piensa-de-lasclases-de-educacion-fisica-y-no-se-atrevea-decirle-a-su-profesor-a/

Bonilla, E., \& Rodríguez, P. (1997). Más allá del dilema de los métodos: la investigación en ciencias sociales. Norma.

Canales, M. (2006). Metodologías de la investigación social. LOM. 
Carcamo-Oyarzun, J., Wydra, G., HernandezMosqueira, C., \& Martinez-Salazar, C. (2017) Actitudes hacia la educación física: Grados de importancia y conformidad según escolares de Chile y Alemania. Una mirada intercultural. Retos. Nuevas tendencias en Educación Física, Deporte y Recreación, (32), 158-162 https://doi.org/10.47197/retos.v0i32.52824

Cárcamo, J. (2012). El profesor de Educación Física desde la perspectiva de los escolares. Estudios Pedagogicos, 38(1), 105-119. https://doi.org/10.4067/s071807052012000100006

Daniel, M., \& Sousa, J. (2010). Percepção das aulas de educação física na visão de alunos da Rede Pública de Juazeiro do Norte - Ce. http://congressos.ifal.edu.br/index.php/con nepi/CONNEPI2010/paper/viewFile/1207/72 4

Dochy, F., Segers, M., \& Dierick, S. (2002). Nuevas vias de aprendizaje y enseñanza y sus consecuencias: una nueva era de evaluación. Revista de Docencia Universitaria, 2(2), 13-30 https://revistas.um.es/redu/article/view/200 51

Galeano, M. (2004). Diseño de proyectos en la investigación cualitativa. Universidad Eafit.

Gaviria, D. (2016). La educación física a través de los ojos y la voz de los estudiantes de grado once de secundaria. Un estudio de caso en la ciudad de Medellín-Colombia. [Tesis doctoral]. Universidad Autónoma de Madrid.

https://repositorio.uam.es/handle/10486/67 4774

Gaviria, D., \& Castejón, F. (2016). La educación física en la voz de los estudiantes de secundaria. Un estudio de caso. Cultura, Ciencia y Deporte, 77(32),

107-118.

http://dx.doi.org/10.12800/ccd.v11i32.711
Goetz, J., \& LeCompte, M. (1988). Etnografía y diseño cualitativo en investigación educativa. Morata.

Jaramillo, L., \& Hurtado, D. (2006). Imaginarios y jóvenes escolarizados. Un encarnamiento deportivizado de la Educación Física en la ciudad de Popayán. Apunts Educación Física y Deportes, (83), 71-76. https://www.redalyc.org/pdf/5516/551656962 009.pdf

Kawashima, L. (2012). Percepções dos Alunos sobre os conteúdos da educação física: Entre o saber instituído e o idealizado. Connectionline, Revista Electrónica do Univag, (8), 72-82. http://dx.doi.org/10.18312/19807347.n8.2012.99

Merriam, S. (1988). Case study research in education a qualitative approach. Jossey-Bass.

Moreno, J., \& Hellín, M. (2007). El interés del alumnado de Educación Secundaria Obligatoria hacia la Educación Física. Revista electrónica de investigación Educativa, 9(2), 1-20. https://redie.uabc.mx/redie/article/view/173/ 301

Murcia, N. (2007). Imaginarios del joven colombiano ante la clase de Educación Física. Educación Física y Deporte, 26(1), 47-57. https://revistas.udea.edu.co/index.php/educ acionfisicaydeporte/article/view/226/156

Murcia, N., \& Jaramillo, L. (2005). Imaginarios del joven colombiano ante la clase de educación física. Revista Latinoamericana de Ciencias Sociales, Niñez y Juventud, 3(2), 175-212. http://www.scielo.org.co/scielo.php?script=s ci_arttext\&pid=S1692-715X2005000200007

Okuda, M., \& Gómez-Restrepo, C. (2005). Métodos en investigación cualitativa: triangulación. Revista Colombiana de Psiquiatría, 34(1), $118-124$

https://www.redalyc.org/pdf/806/80628403 009.pdf

Piéron, M. (1999). Para una enseñanza eficaz de las actividades físico-deportivas. Inde. 
Phillips, S., Marttinen, R., Mercier, K., \& Gibbone, A. (2020). Middle School Students' Perceptions of Physical Education: A Qualitative Look. Journal of Teaching in Physical Education 40(1), 30-38. https://doi.org/10.1123/jtpe.20190085

Schiffman, H. (2004). Sensación y percepción: un enfoque integrador. El manual moderno.

Sözen, H., \& Korur, E. N. (2019). "A physical education teacher is like...": Examining Turkish students' perceptions of physical education teachers through metaphor analysis. International Electronic Journal of Elementary Education, 12(2), 183-188. https://doi.org/10.26822/iejee.2019257665
Strauss, A., \& Corbin, J. (2002). Bases de la investigación cualitativa: Técnicas y procedimientos para desarrollar la teoría fundamentada. Universidad de Antioquia. https://diversidadlocal.files.wordpress.com/ 2012/09/bases-investigacion-cualitativa.pdf

Taylor, S., \& Bogdan, R. (1987). Introducción a los métodos cualitativos de investigación. Paidós.

Yin, R. (1984). Case study research: design and methods, applied social research methods. Sage.

Dirección para correspondencia

Sarita Moreno Pérez

Licenciada en Educación Física

Universidad de Antioquia.

Medellín, Colombia.

Dirección postal: 050035

ORCID ID: https://orcid.org/0000-0001-8464-717X

Contacto:

sarita.moreno@udea.edu.co

Recibido: 12-11-2020

Aceptado: 09-06-2021

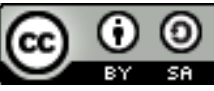

